\title{
Pressure Measurements in Jets Diffusion using a Developed Hot-Wire Static Pressure Sensor*
}

\author{
Motoaki KIMURA** and Norimasa MIYAGI*** \\ **Department of Mechanical Engineering, College of Science \& Technology, Nihon University, \\ 1-8-14 Kanda Surugadai, Chiyoda-ku, Tokyo 101-8308, Japan \\ E-mail: kimura@mech.cst.nihon-u.ac.jp \\ ***Nihon University Junior College Funabashi Campus, \\ 7-24-1 Narashinodai Funabashi, Chiba 274-8501, Japan
}

\begin{abstract}
The aim of this experimental study is to investigate the diffusion of jets, with particular attention focused on the relationship between the static pressure and the stream wise mean velocity on the development of a two-dimensional air jet. A jet with three injection velocities $(11.7,23.3$ and $35.0 \mathrm{~m} / \mathrm{s})$ was generated in a two-dimensional wind tunnel. The velocity distributions were measured by an $\mathrm{X}$-type hot-wire anemometer. The static pressure distributions were measured by a static pressure probe developed in our laboratory, which incorporates a hot-wire anemometer. The probe is designed to be able to measure the mean static pressure and pressure fluctuation simultaneously. The sensitivity is $92.3 \mathrm{mV} / \mathrm{Pa}$. The frequency response is flat from $16 \mathrm{~Hz}$ to $2.5 \mathrm{kHz}$. As a result of the experiment, it was found that negative static pressure exists in the turbulent shear layer. It is considered that the entrainment process from the negative static pressure by the vortex structure motion of the turbulent shear layer.
\end{abstract}

Key words: Jet, Pressure Measurement, Pressure Sensor, Entrainment, Diffusion

\section{Introduction}

The need for suitable instrumentation to measure mean and fluctuating pressures in turbulent shear flows has been self-evident for many years. The possibility as well as the limitations of direct fluctuating pressure measurements in turbulent flows have been discussed by Fuchs ${ }^{(1)}$ and Willmarth ${ }^{(2)}$. The insertion of pressure probes into the flow causes several error mechanisms, namely, acoustic contamination, wind-noise, affected sensitivity, acceleration response, a resolution error, error due to fluctuating cross flow and response to axial velocity fluctuation. Some pressure measuring techniques in unsteady flows have been developed, though they are subject to many potential sources of error.

Transducers used in high response pressure measuring probes can be classified into three types: the condenser microphone type ${ }^{(3)-(5)}$, the ceramic piezoelectric type ${ }^{(6)}$ and the hot-wire type ${ }^{(7)-(9)}$. Arndt and Nilsen ${ }^{(3)}$ measured the fluctuating pressure in the mixing zone of a round jet using a microphone pressure probe which consisted of a static pressure tube and a 1/8 inch condenser microphone. Sami, Carmody and Rouse ${ }^{(6)}$ developed a pressure probe for the measurement of pressure fluctuations using a ceramic piezoelectric tube. Remenyik and Kovasznay ${ }^{(7)}$ developed an orifice-hot-wire probe whose sensing element in the pressure probe was a hot-wire spanning the cross section of the hole near an orifice in a wall. This probe measured wall pressure fluctuations. Spencer and Jones ${ }^{(8)}$ developed a

${ }^{*}$ Received 5 Dec., 2008 (No. 08-0873) [DOI: 10.1299/jfst.4.256] 
bleed-type static pressure transducer using a hot-film, and Nasseri and Nitsche ${ }^{(9)}$ developed a suction-type total pressure probe using a hot-wire. Both these pressure probes used a slow bias flow around the hot-wire with bleed air or suction to render the hot-wire response linear to pressure fluctuation. Although many studies of the effectiveness of the respective probes in measuring pressure fluctuation have been carried out, no studies have ever aimed to develop a probe capable of measuring the mean static pressure and the pressure fluctuation simultaneously. One of the important advantages of the devices mentioned above is that the mean static pressure and the static pressure fluctuation can be measured simultaneously.

This experimental study aims at developing and calibrating a suitable hot-wire static pressure probe, and then using this probe to investigate the diffusion of jets. Particular attention is focused on the relationship between the static pressure and the stream wise mean velocity on the development of a two-dimensional air jet. A jet with three injection velocities $(11.7,23.3$ and $35.0 \mathrm{~m} / \mathrm{s})$ was generated in a two-dimensional wind tunnel and used as the basis of this investigation.

\section{Nomenclature}

$\begin{array}{ll}b: & \text { half width of jet } \\ f: & \text { frequency } \\ h: & \text { nozzle height } \\ P: & \text { mean pressure } \\ P i: & \text { pressure of the air supply (constant) } \\ P o: & \text { atmospheric pressure } \\ P s: & \text { mean static pressure } \\ P s i: & \text { indicated mean static pressure } \\ P s^{\prime}: & \text { fluctuating pressure (r.m.s.) } \\ P s i{ }^{\prime}: & \text { indicated fluctuating pressure (r.m.s.) } \\ U: & \text { mean velocity } \\ U m: & \text { maximum mean velocity in the cross section } \\ u^{\prime}: & \text { stream wise fluctuating velocity (r.m.s.) } \\ v^{\prime}: & \text { lateral fluctuating velocity (r.m.s.) } \\ x: & \text { stream wise coordinate } \\ y: & \text { cross-sectional coordinate } \\ \theta: & \quad \text { yaw angle } \\ \rho: & \text { density of air }\end{array}$

\section{Hot-Wire Static Pressure Probe}

\subsection{Principles of Hot-Wire Static Pressure Probe Design and Operation}

The hot-wire static pressure probe is a transducer that converts pressure difference to velocity, from which an output in electrical form may be generated. Similarly, constant temperature hot-wire anemometers are used to convert a velocity to electrical form. The static pressure probe developed in this investigation consists essentially of a static pressure tube and a hot-wire spanning the cross section of the static pressure tube (Fig. 1). The static pressure tube incorporates several small static pressure holes. The tube is connected to an air supply system that supplies air at a constant pressure $P i$. The constant pressure $P i$ is slightly greater than atmospheric pressure, $P o$, to create a bias flow at the static pressure holes through the hot-wire section. Therefore the pressure difference $\mathrm{Pi}-\mathrm{Po}$ can be maintained at a constant value with this device. The velocity of the air flow from the static pressure holes is very low. Therefore, the effect of this flow is negligibly small in comparison with the free stream flow being measured. The air velocity at the hot-wire is 
Po: Atmospheric pressure

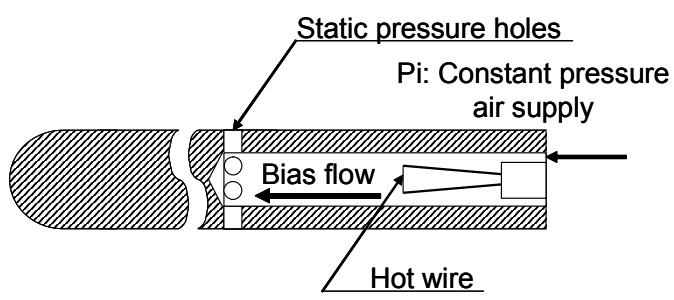

Fig. 1 Cross section of static pressure tube with hot-wire.

driven by the pressure difference, $P i-P s$, between the static pressure, $P s$, and the pressure of the air supply, $P i$. When pressure fluctuations are incident into the static pressure holes, induced velocity fluctuation is uniquely determined and a voltage is produced.

There are two important characteristics of hot-wire static pressure probes. The first is that the mean static pressure and the static pressure fluctuation can be measured simultaneously. The second is that the probe is not affected by the free stream flow or any changes in the physical properties (thermal conductivity) of the flow, because the sensing hot-wire is always in the air bias flow. The probe shape is designed to minimize the affect of the probe on the flow during operation.

\subsection{Probe Structure and Systems}

The system used in this study to measure static pressure is shown diagrammatically in Fig. 2. This system consists of a constant pressure air supply system, a Prandtl-type static pressure tube, and a constant temperature hot-wire anemometer. The air supply system comprises an air pump, a pressurizing water tank, a desiccator, a pressure stabilizing tank, a regulation valve and a manometer type pressure gauge. The pressure may be maintained at a constant value by maintaining the water column at a constant height. Very small constant pressures are conveyed to the $0.01 \mathrm{~m}^{3}$ volume tank. The tank has a leak valve of area 380 $\mathrm{mm}^{2}$ when fully open. The pressure in the pressure tank is regulated automatically, and maintained at a constant value, by the leak valve.

The static pressure probe consists of a static pressure tube and a hot-wire spanning the

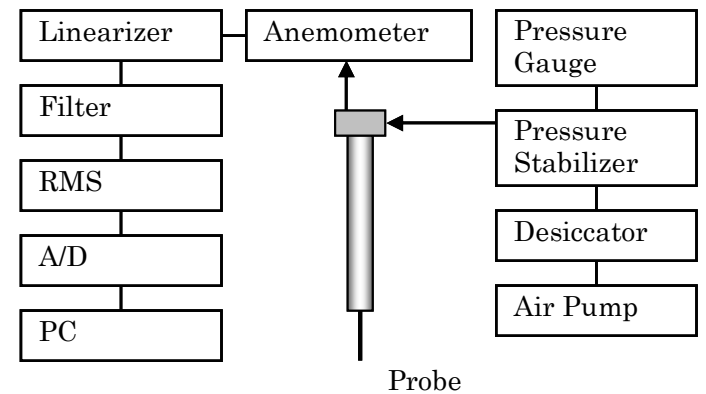

Fig. 2 Static pressure measuring system.

Po: Atmospheric pressure

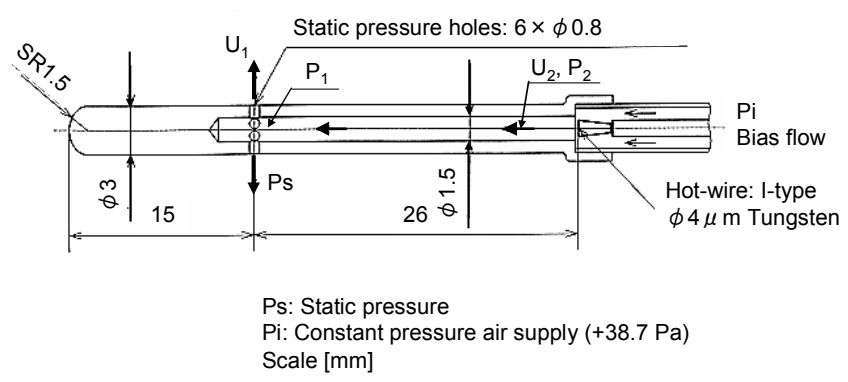

Fig. 3 Dimensions of the static pressure probe. 
cross section of the internal portion of the tube, as shown in Fig. 3. The static pressure tube is of outside diameter $D_{1}=3 \mathrm{~mm}$ and internal diameter $D_{2}=1.5 \mathrm{~mm}$. The six evenly spaced static pressure holes of diameter $0.8 \mathrm{~mm}$ are drilled radially at a distance of $L_{1}=15 \mathrm{~mm}$ from the probe nose. The distance $L_{2}$ between the static pressure holes and the face internal to the sleeve, which enables connection with the hot-wire section, is $26 \mathrm{~mm}$. The internal diameter of the static pressure tube $D_{2}$ influences the hot-wire sensitivity. The distances $L_{1}$ and $L_{2}$ influence the pressure distribution around the static pressure tube ${ }^{(10)}$. The probe dimensions were determined by experimental trial and error. The hot-wire was sourced from a commercially available probe manufactured by TSI (Model 1260A-1.5, wire diameter: 4 $\mu \mathrm{m}$, wire length: $1.4 \mathrm{~mm}$ ). This tungsten hot-wire is located at the cross section of the static pressure tube. The bridge circuit and the amplifier of the anemometer system (Kanomax Model 1010) were also incorporated. During operation, the hot-wire anemometer is maintained at a constant-temperature; the over heat ratio $(R w / R g)$ is 1.5 , where $R w$ is the working electrical resistance of the wire, and $R g$ denotes the electrical resistance of the wire at the gas temperature. It is important that the bias flow is kept at a suitable level within an allowable pressure fluctuation, because of the exact pressure measurement. The necessary and just very small pressures for slow bias flows was determined by comparing the test probe with the standard static pressure tube. The optimum pressure for the bias flow was determined to be $38.7 \mathrm{~Pa}$. At this pressure, the bias flow velocities are estimated at $U_{1}=0.38$ $\mathrm{m} / \mathrm{s}$ at the static pressure holes and $U_{2}=0.66 \mathrm{~m} / \mathrm{s}$ at the hot-wire; these velocities were measured by a soap membrane flow meter. The Reynolds number, $R e=d U_{2} / v$, at the hot-wire is $0.17\left(U_{2}=0.66 \mathrm{~m} / \mathrm{s}, d=4 \mu \mathrm{m}, v=15.5 \mathrm{~mm}^{2} / \mathrm{s}\right)$. Thus, according to $\operatorname{King}^{\prime} \mathrm{law}^{(11)}$, the Reynolds number is in a suitable range for hot-wire anemometry,.

\subsection{Calibration}

We now describe the calibration of sensitivity, frequency response, and directivity in the flow (yaw angle characteristics) for the probe described above. The calibration methods were devised by the authors.

\subsubsection{Sensitivity}

The sensitivity calibration apparatus is shown in Fig. 4. The test probe is set in a pressure tank of diameter $300 \mathrm{~mm}$, and depth of $350 \mathrm{~mm}$. The pressure tank can be pressurized from positive pressures to negative pressures (relative to atmospheric pressure). The tank is pressurized by the bias flow of the probe itself to generate positive pressures. The vacuum pump evacuates air from the pressure tank to produce pressure below atmospheric pressure. The pressure in the tank is kept constant by the regulation valve, which is determined by an inclined manometer with a precision of $1 \mathrm{~Pa}$. The pressure $\mathrm{Pi}$ for the bias flow is $38.7 \mathrm{~Pa}$ as explained in the previous section (2.2 Probe Structure and Systems). The relationship between the pressure difference $P s-P o$ and the output voltage of

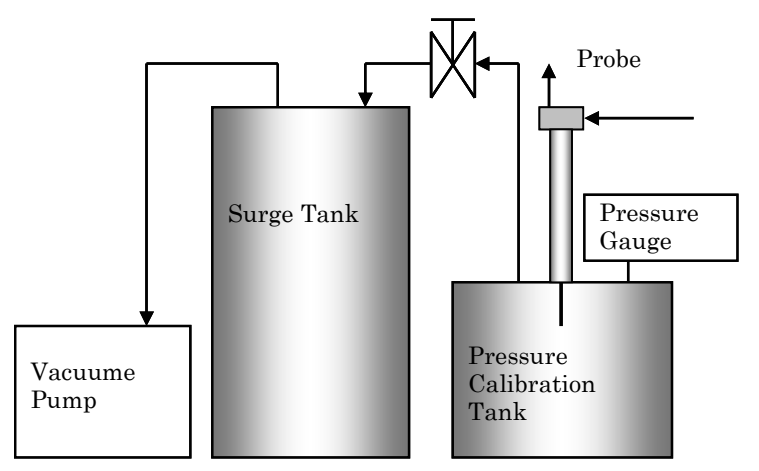

Fig. 4 Sensitivity calibration apparatus. 
the DC amplifier, $E$, is shown in Fig. 5. For the purposes of hot-wire anemometry it is convenient and conventional to write the relation between velocity and output in the form $E^{2}=A+B U w^{0.5}$ in accordance with King's law, where $U w$ is the velocity of the flow passing the hot-wire, and $A$ and $B$ are experimental constants. The diameter $\left(D_{2}=1.5 \mathrm{~mm}\right)$ was determined by experiment. In the case that the diameter is larger than $D_{2}$, the output $E$ is distorted. In the case that the diameter is less than $D_{2}$, the output is unsteady. In the case of the determined diameter $\left(D_{2}=1.5 \mathrm{~mm}\right)$ the value of the probe output voltage indicates only a single static pressure. The level of the probe output for a pressure change is sufficient to measure the change. The probe output voltage $E$ is linearized by the linearizer (Fig. 2). In practice, the linearized probe sensitivity is $92.3 \mathrm{mV} / \mathrm{Pa}$, which is linear within $0.3 \%$ of the pressure measurement limits $[P S-P o=-80 \mathrm{~Pa}$ to $+35 \mathrm{~Pa}$, ref. Atmospheric pressure, $P o]$. The dynamic range of the combined static pressure probe and amplifier system is characterized by defined upper and lower limits. The upper limits are generally specified for degree of distortion, while the lower limits are given as noise levels for the various bandwidths of the associated indicating equipment. The lower limit of the static pressure probe and amplifier combination is $80.7 \mathrm{~dB}$ [ref. $2 \times 10^{-5} \mathrm{~N} / \mathrm{m}^{2}$ ]. We could not measure the upper limit, due to the lack of a high-powered loudspeaker system.

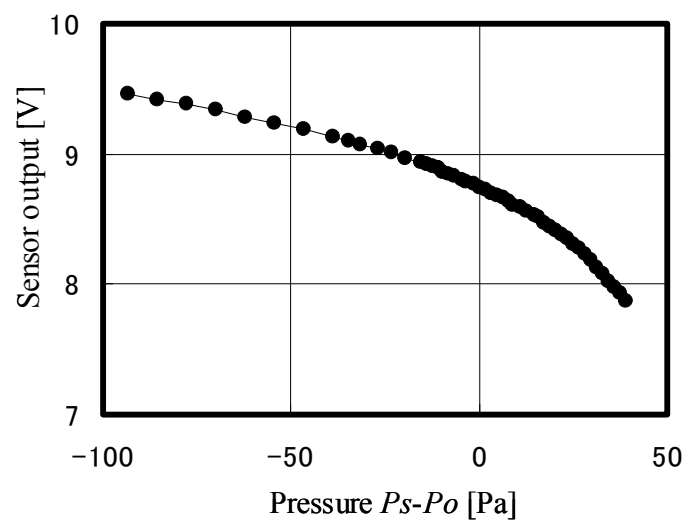

Fig. 5 Pressure calibration curve.

\subsubsection{Frequency Response}

Since the flows at the static pressure holes and around the hot-wire are unsteady, the unsteady effect must be considered for the velocity and the pressure measurements. With reference to Fig. 3, which shows the static pressure probe labeled with the relevant velocity and pressure variables, the gas density $\rho$ is considered to be constant because only very small changes in pressure occur. Assuming that the pressure drop in the tube is negligible, then

$U_{1}=\left[2\left(P_{1}-P S\right) / \rho\right]^{0.5}$

where $P_{1}$ is the pressure at the static pressure holes inside the probe and $P S$ is the static pressure at the static pressure holes outside the probe. The continuity relation for this situation is

$Q=\rho N U_{1} \pi d^{2} / 4=\rho U_{2} \pi D^{2} / 4$

where $N$ is the number of the static pressure holes, $d$ is the static pressure hole diameter, $D$ is the internal diameter at the hot-wire, and $U_{2}$ is the velocity at the hot-wire. The pressure drop is negligible, because of the very small velocity; that is, 
If the static pressure changes rapidly by $\Delta P$, the pressure change $\Delta P$ propagates at the speed of sound from the section containing the static pressure holes to the section containing the hot-wire. The velocity change $\Delta U_{1}$ occurs after the sound wave front passes,

$$
\Delta U_{1}=\left[2\left(P_{1}-P s-\Delta P\right) / \rho\right]^{0.5}-U_{1}
$$

The hot-wire senses the velocity change due to the pressure change. The propagation time from the section containing the static pressure holes to the hot-wire section is $\Delta t=\Delta x / a$, where " $a$ " is the speed of sound in the medium. The time $\Delta t$ is the delay time of the static pressure probe. The delay time is $76 \mu \mathrm{s}\left[\Delta x=26 \times 10^{-3} \mathrm{~m}, a=340 \mathrm{~m} / \mathrm{s}\right]$ in the case of the static pressure probe. Therefore, the theoretical frequency range is less than $c=1 / \Delta t[12.6 \mathrm{kHz}]$. The practical frequency response is less than the theoretical frequency range.

The frequency response characteristic of the probe was determined by a probe calibration system comprising a B\&K type 4138 1/8 inch standard condenser microphone, a

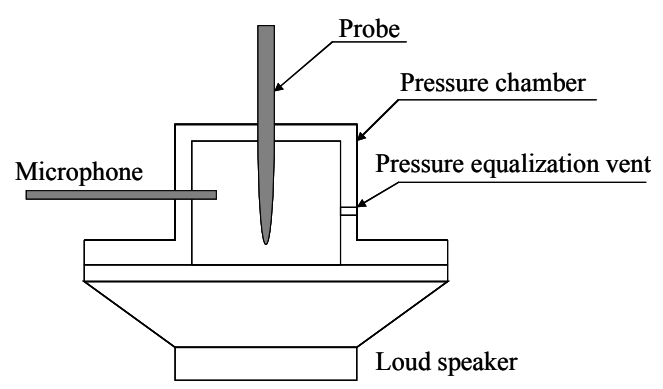

Fig. 6 Frequency response measurement apparatus.

B\&K type 2618 microphone preamplifier, a frequency generator, a loudspeaker and a pressure chamber. The general arrangement of this system is shown diagrammatically in Fig. 6. The sound source (loudspeaker) is powered by the power amplifier via the frequency generator. The probe, the sound source and the $1 / 8$-inch microphone are placed in the chamber such that the probe and the microphone are in the radiated sound field of the sound source. The sound pressure in front of the probe and the microphone must be the same and constant. Therefore, the two components used for this calibration were placed in close proximity. The sound pressure level output of the loudspeaker was set at $104 \mathrm{~dB}$ [ref. $2 \times 10^{-5}$ $\mathrm{N} / \mathrm{m}^{2}$ ] and the frequency was varied from $16 \mathrm{~Hz}$ to $2.5 \mathrm{kHz}$. The frequency response error is compensated for electrically by a band-rejection filter, a band-pass filter and a frequency booster. By the use of these devices, the final overall compensated response of the pressure probe was flat within $1.2 \mathrm{~dB}$ from $16 \mathrm{~Hz}$ to $2.5 \mathrm{kHz}$ as shown in Fig. 7. It can be considered

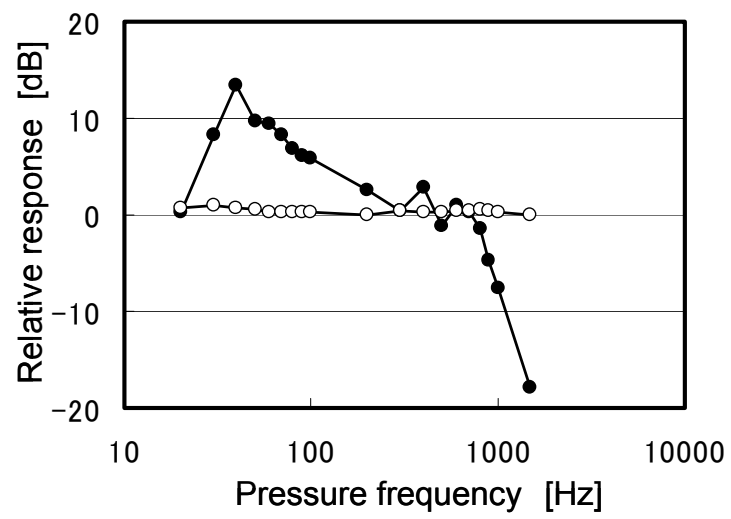

Fig. 7 Frequency response ( natural response: compensated response: $\bigcirc$ ). 
that the phase difference is negligibly small for the measured frequency due to the short distance between the static pressure holes and the hot-wire, because the propagation time of the pressure wave from the static pressure holes to the hot-wire is $76 \mu$ s. The one-to-one correspondence is kept under the frequency range of the static pressure probe. This demonstrates that the static pressure probe can measure the pressure in unsteady flow under the frequency range of the static pressure probe.

\subsubsection{Yaw Angle Characteristics}

The test probe was set in the constant flow of the potential core region of a square nozzle jet. The dimension of the nozzle is $200 \times 200 \mathrm{~mm}$. A screen was located on the nozzle exit in order to keep the isotropic turbulence level at the test section constant. The screen mesh interval is $10 \mathrm{~mm}$, and the cross sectional size of the rods which make up the mesh is $2 \times 2 \mathrm{~mm}$. Under these operating conditions, the measured stream-wise turbulence level $u^{\prime} / U \times 100$ was $5 \%$ and the transverse turbulence level $v^{\prime} / U \times 100$ was $4 \%$. The test probe can be tilted from plus to minus 30 degrees of yaw away from the flow direction around the center of the static pressure holes of the test probe. The mean static pressure error, $(P s i-P s) /\left(\rho U^{2} / 2\right) \times 100$, is presented for the case in which flow velocity is $10 \mathrm{~m} / \mathrm{s}$. Fig. 8 shows the yaw angle characteristics. The mean static pressure error is less than $4 \%$ between 0 and 10 degrees of yaw. The fluctuating static pressure error, $\left(P^{\prime} s i-P{ }^{\prime}\right) /\left(\rho U^{2} / 2\right) \times 100$, is presented in the same condition. The fluctuating static pressure error is less than $1 \%$ between 0 and 10 degrees of yaw. The static pressure error tendency of this probe is almost the same as the standard Prandtl-type static pressure tube ${ }^{(12)}$, because the nose shape of this probe is same as that of the Prandtl tube.

\section{Pressure Measurements of a Two-Dimensional Jet}

\subsection{Experimental Setup}

As an example of an actual measurement, the probe was used to measure the static pressure in the development of a rectangular jet. The rectangular jet was generated in a two-dimensional rectangular wind tunnel that utilizes five screens and one honeycomb for turbulence reduction in the wind tunnel (Fig. 9). The dimensions of the rectangular nozzle exit are $h=13 \mathrm{~mm}$ (height) by $300 \mathrm{~mm}$ (width). The contraction ratio and the exit aspect ratio are 52 and 23, respectively. At these operating conditions, the measured stream-wise turbulence level $u$ ' $/ U \mathrm{x} 100$ is about $1.9 \%$ at the nozzle exit. The exit velocities are $11.7,23.3$ and $35.0 \mathrm{~m} / \mathrm{s}$. Therefore, the exit Reynolds numbers based on the nozzle hight $h(13 \mathrm{~mm})$ are $1.0 \times 10^{4}, 2.0 \times 10^{4}$ and $3.0 \times 10^{4}$, respectively. Mean static pressure and fluctuating pressure were measured by the static pressure probe. An X-type hot-wire sensor (Kanomax 0252-T5) and anemometers (Kanomax Model 1010, 1013, 1008, 1075 and 1017) were used

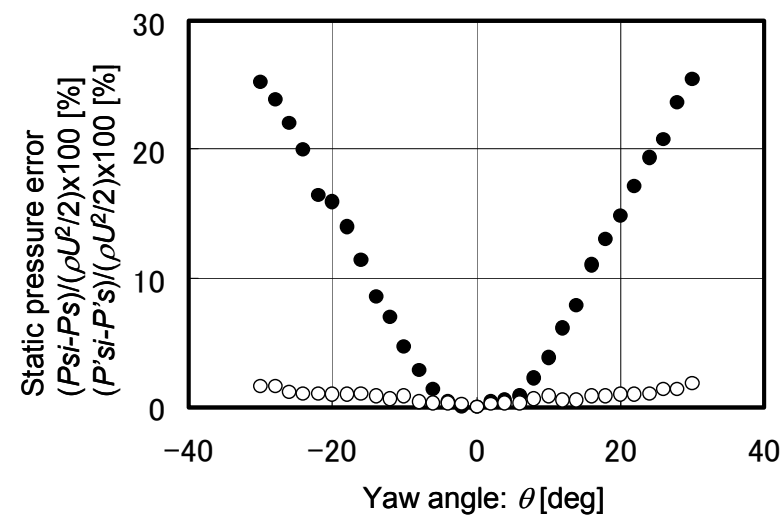

Fig. 8 Yaw angle Characteristics on mean static pressure $(\mathbf{O})$ and fluctuation static pressure $(\bigcirc)$. 
to measure velocity. The smoke-wire method was used to visualize the two-dimensional jet. Nichrome wire was installed along both sides $(300 \mathrm{~mm})$ of the nozzle to generate oil smoke. The smoke was illuminated by a lamp, and photographs were taken.

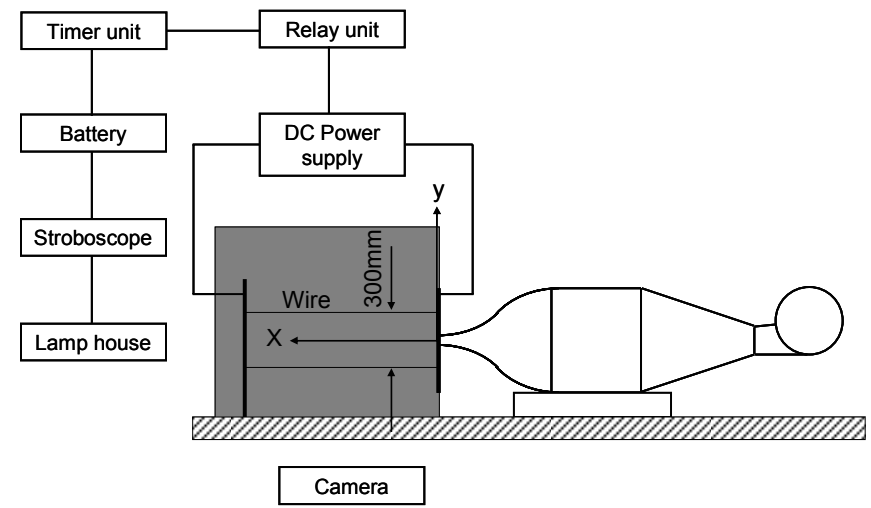

Fig. 9 Experimental setup of two dimensional jets.

\subsection{Results and Discussion}

Figure 10 shows the visualization photograph of the jet diffusion process using the smoke wire method. The delay time after smoking is $300 \mathrm{~ms}$. We can observe the jet diffusion structure, potential core region, fully developed region, vortex structure and entrainment flow. For example, the smoke flows straight from the outer regions to the center in the locations of plus or minus $y / h=10$. It is thought that this area exhibits little fluctuation because the static pressure fluctuation is low. Closer to the jet center, around plus or minus $y / b=1$, fluctuation occurs, and the smoke is rolled up in the jet and drifts. The neighborhood where fluctuation occurs is regarded as a free shear layer. The vortex occurring in the shear layer is intermittent. It is therefore thought that the neighboring air is rolled up in the jet intermittently. A large-scale structure is formed in the free shear layer. The flow stagnation and entrainment flow are observed from the flow visualization. The entrainment flow stagnates on the surface where the vortex structure touches the surrounding atmosphere because the pressure rises. The entrainment flow is rolled between vortex structures because the pressure decreases. It is observed that the stream wise wave pattern of the smoke which forms with the flow stagnation and rolling flow due to the moving the vortex structure, and the lateral wave pattern of the smoke which forms with the three-dimensional structure.

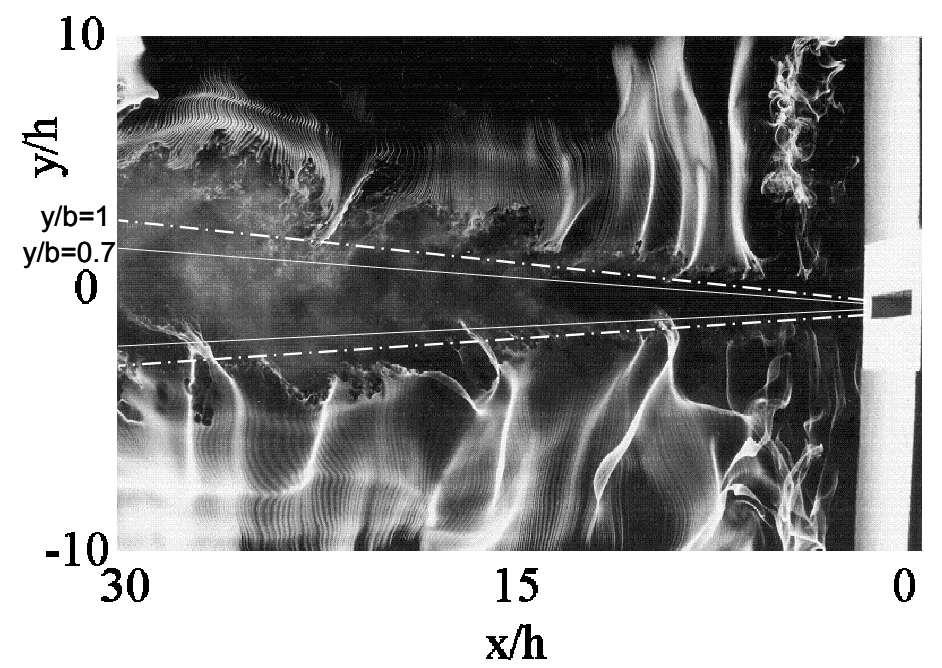

Fig. 10 Visualization of the jet using smoke wire $\left(R e=3.0 \times 10^{4}\right)$. 


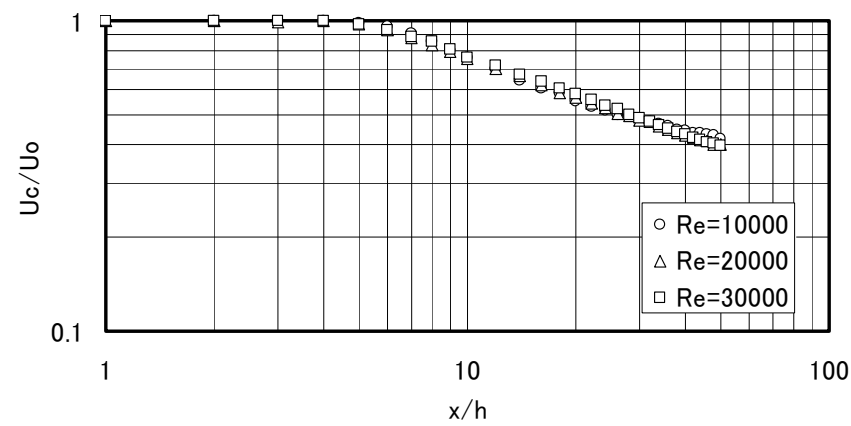

Fig. 11 Velocity decay of the jet center $\left(R e=3.0 \times 10^{4}\right)$.

The velocity decay of the jet center is shown in Fig. 11. The potential core region is about 4 nozzle heights in length $(x / h=4)$. There is a fully developed region after the transitional region, the slants of the velocity decrement decrease along the slope $U c / U o=c(X / h)^{-1 / 2}$ in the range $x / h=10-50$. The mean velocity distributions of the rectangular jet are shown in Fig. 12, where $y$ is the cross-section coordinate, $x$ is the distance from the nozzle exit, $U m$ is the center-line velocity and $b\left(b=c_{1}+c_{2} x\right)$ is the half width of the jet at which the velocity $U$ is half of its center-line value. If we plot $U / U m$ against $y / b$ for various sections, they all fall on a single curve as shown in Fig. 12, thereby showing that the velocity distribution is indeed similar in the fully developed region. The velocity profiles at different sections that could be superimposed in this manner are said to be similar. The fluctuating velocity distributions of the jet are shown in Fig. 13. The peak of the velocity fluctuation distribution is observed at the free shear layer. If we plot $u$ '/Um against $y / b$ for various cross sections in the fully developed region, they all fall on a single curve as shown

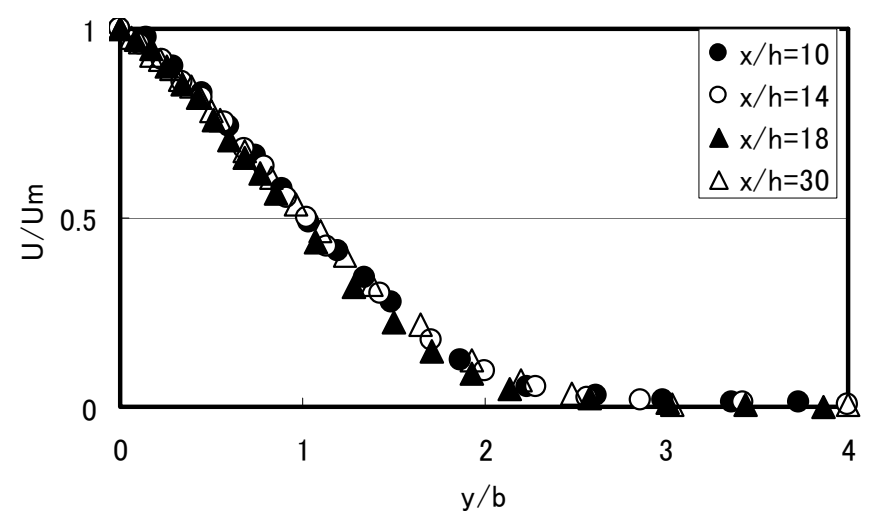

Fig. 12 Mean velocity distributions $\left(R e=3.0 \times 10^{4}\right)$.

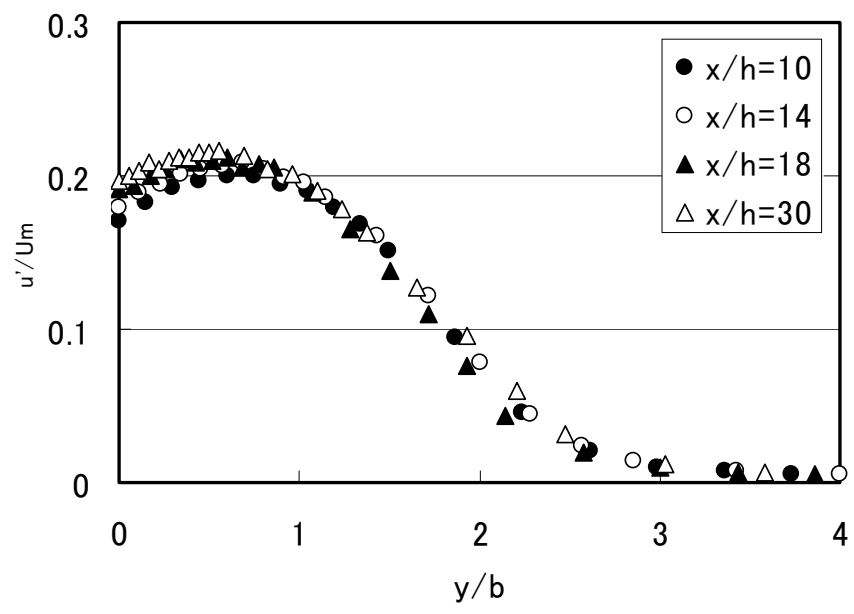

Fig. 13 Fluctuation velocity distribution $\left(\operatorname{Re}=3.0 \times 10^{4}\right)$. 
in Fig. 13, thereby showing that the fluctuating velocity distribution is similar in the fully developed region.

The mean static pressure distributions at various cross sections are shown in Fig. 14. The mean static pressure distributions at these cross sections have negative values. Negative pressure (from -7 to $-12 \mathrm{~Pa}$ ) occurs in the jet center zone. The minimum negative values occur at the center of the free shear layer. In the outer range, $y / b>4$, the negative pressure decreases in lower than measurement limit. In the downstream region, the negative pressure decreases. The pressure fluctuation distributions are shown in Fig. 15. The peak of the pressure fluctuation distribution is observed at the center of the free shear layer. It may be considered that the negative static pressure results from the vortex that occurs in the free

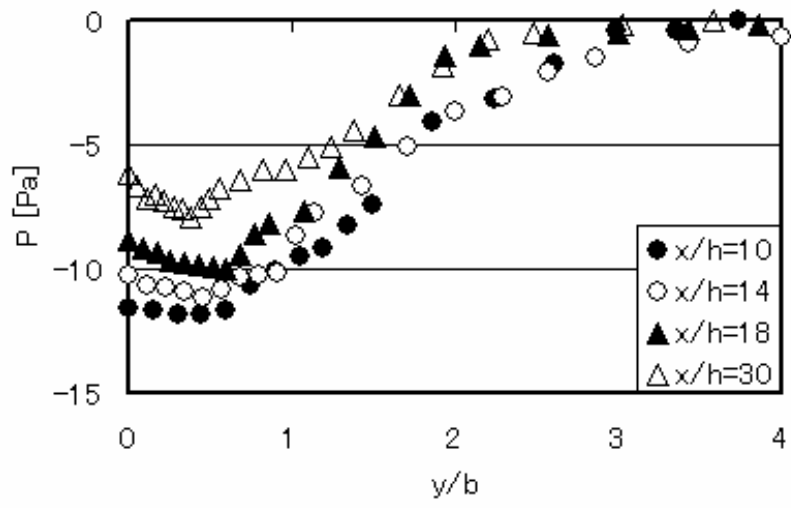

Fig. 14 Mean pressure distributions $\left(R e=3.0 \times 10^{4}\right)$.

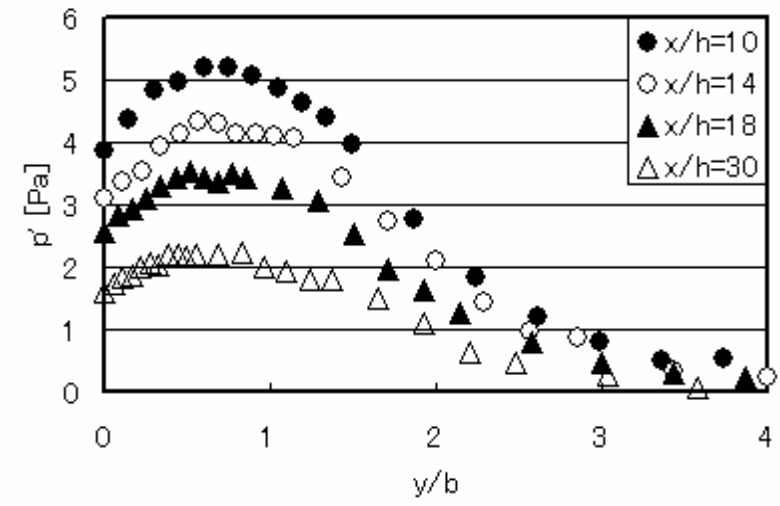

Fig. 15 Fluctuation pressure distributions $\left(R e=3.0 \times 10^{4}\right)$.

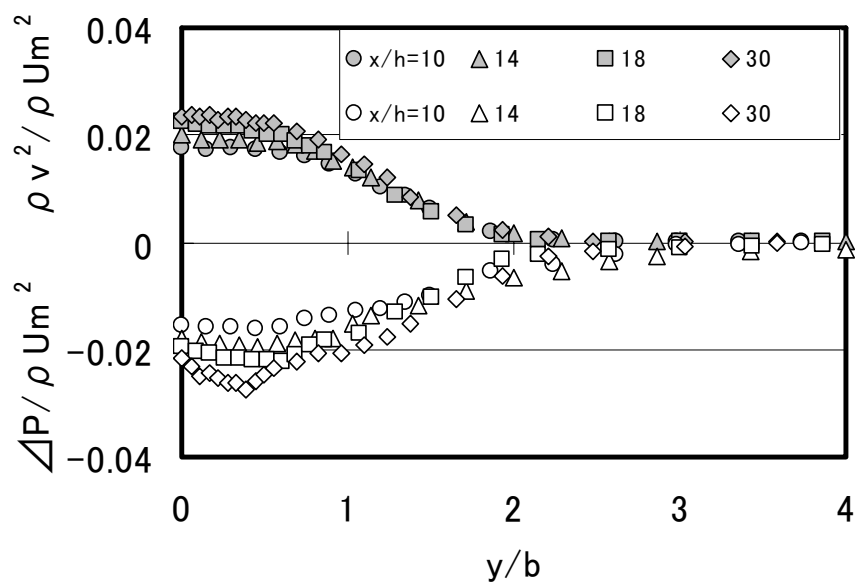

Fig. 16 Lateral Reynolds normal stress and mean pressure distributions $\left(R e=3.0 \times 10^{4}\right)$. 
shear layer.

A comparison between the mean static pressure distribution and the Reynolds normal stress distributions are shown in Fig. 16; similarities between mean static pressure distributions can be clearly observed. In the figure, " $\rho U m^{2}$ " is the momentum at the center of the cross section. The figure shows that the mean static pressure and the fluctuating pressure distributions are indeed similar in the fully developed region. The peak of these distributions is observed at the center of the free shear layer center $(y / b=0.75)$.

Under the restrictions placed on the flow (two-dimensional, incompressible, steady on average), the appropriate lateral equation of motion, with simplified notation, is

$0=-\frac{1}{\rho} \frac{\partial}{\partial y}\left(P+\rho v^{\prime 2}\right)$

Dimensional analysis of the magnitudes of the various terms in the equation led Townsend $^{(13)}$ to the conclusion that the lateral gradients of the turbulent Reynolds normal stress and static pressure are the dominant terms and that the integrated form of the equation may be written

$$
P+\rho v^{\prime 2}=P_{\infty}
$$

where $P_{\infty}$ is a function of stream-wise distance and is equal to the static pressure outside the flow. The lateral Reynolds normal stress matches the mean static pressure in magnitude and the tendency on that section.

It is thought that there is a relation between the jet diffusion and the negative pressure in the jet as follows. From Fig. 14 the mean static pressure of the entire jet flow becomes negative pressure from the atmospheric pressure. A vortex structure by the flapping motion of the two dimensional jet grows up from the visualization as shown in Fig. 10. On the surface where the vortex structure touches the surrounding atmosphere, because the rotation of the vortex structure is the opposite direction against the jet flow, the velocity becomes low, and pressure rises. On the surface where the vortex structure touches the jet center, because the rotation of the vortex structure is the same direction with the jet flow, the velocity becomes high, and pressure decreases. The velocity fluctuation is generated due to passing the vortex structure. The velocity fluctuation induces the pressure fluctuation. From Fig. 13 and Fig. 15 the position in which the velocity fluctuation and the pressure fluctuation become the maximum is the location of around $y / b=0.75$.

\section{Conclusion}

A static pressure probe was developed using a hot-wire anemometer. The probe was designed to measure the time mean static pressure and pressure fluctuations simultaneously. Experiments were carried out to determine the optimum dimensions of the probe, the most suitable operating conditions and the probe characteristics. The probe was used to measure the static pressure in the development of a two-dimensional jet. The following conclusions were reached:

The probe sensitivity is $92.3 \mathrm{mV} / \mathrm{Pa}$, which is linear within $0.3 \%$ between the pressure measurement limits [ $-80 \mathrm{~Pa}$ to $+35 \mathrm{~Pa}$, ref. atmospheric pressure]. The overall frequency response of the probe is flat within $1.2 \mathrm{~dB}$ from $16 \mathrm{~Hz}$ to $2.5 \mathrm{kHz}$. The mean static pressure error is less than $4 \%$ within plus or minus 10 degrees of yaw, and the fluctuating static pressure error is less than $1 \%$ within plus or minus 10 degrees of yaw. In this practical investigation, reasonable characteristics were ensured for very small static pressures.

Owing to the experimental results of mean velocity, fluctuating velocity and static 
pressure distributions in the shear layer of a two-dimensional jet, it is concluded that a negative static pressure region exists in the turbulent shear layer, and the relation between the jet diffusion and the negative static pressure distribution is discussed.

\section{Acknowledgments}

The authors wish to express their indebtedness to Professors Hajime Fujita and Hikaru Miura of Nihon University for their valuable suggestions. The authors would also like to thank Mr. Noriaki Kitahara, Mr. Mitsushi Maeyama, Mr. Tomokazu Harada, Mr. Masaru Tokuda, Mr. Kaoru Haishi, Mr. Takashi Kurosawa, and Mr. Noritaka Kumagai for their help in this investigation.

\section{References}

(1) Fuchs, H. V., Measurement of Pressure Fluctuations within Subsonic Turbulent Jets, Journal of Sound and Vibration, Vo1. 22, No. 3 (1972), pp. 361-378.

(2) Willmarth, W. W., Unsteady Force and Pressure Measurements, Annual Review of Fluid Mechanics, Vo1. 3 (1971), pp. 147-170.

(3) Arndt, R. E. A. and Nilsen, A.W., On the Measurement of Fluctuating Pressure in the Mixing Zone of a Round Jet, ASME Fluid Engineering Division, Paper No. 71-FE-31 (1971).

(4) Maekawa, T., Kawamura, M., Kobashi, Y. and Kono, N., Pressure Fluctuation Measuring Apparatus with Condenser Microphone, Proceedings of the Fifteenth Japan National Congress for Applied Mechanics, (1966), pp. 159-163.

(5) Shirahama, Y. and Toyoda, K., Development of Probe to Measure Static-Pressure Fluctuations (Application to The measurements of Jets), Transaction of the Japan Society of Mechanical Engineers B, Vol. 59, No. 567 (1993), pp. 3381-3387.

(6) Sami, S., Carmody, T. and Rouse, H., Jet Diffusion in the Region of Flow Establishment, Journal of Fluid Mechanics, Vo1. 27, Part 2 (1967), pp. 231-252.

(7) Remenyik, C. J. and Kovasznay, L. S. G., The "Orifice-Hot-Wire" Probe and Measurements of Wall Pressure Fluctuations, Proceedings of the 1962 Heat Transfer and Fluid Mechanics Institute (1962), pp. 76-88.

(8) Spencer, B.W. and Jones, B. G., A Bleed-Type Pressure Transducer for In-Stream Measurement of Static Pressure Fluctuations, The Review of Scientific Instruments, vo1. 42, No. 4 (1971), pp. 450-454.

(9) Nasseri, M. and Nitsche, W., Development of a Probe for Measuring Pressure Diffusion, Proceedings of Eighth Symposium on Turbulent Shear Flows, (1991), pp. (4-4-1)-(4-4-6).

(10) Hubbard, C. W., Investigation of Errors of Pitot Tubes, Transactions of the ASME, Vol. 61 (1939), pp. 477-506.

(11) Hinze, J. O. Turbulence (second edition) (1975), McGraw-Hill, pp. 85-119.

(12) Krause, L. N. and Gettleman, C. C., Considerations Entering into the Selection of Probes for Pressure Measurement in Jet Engines, ISA Proceedings, Vo1. 7 (1952), p.134.

(13) Townsend, A. A., The Structure of Turbulent Shear Flow, (1976), Cambridge University Press, pp. 130-133. 